Pacific Journal of Mathematics

ON OPERATOR ALGEBRAS CONTAINING CYCLIC BOOLEAN 


\title{
ON OPERATOR ALGEBRAS CONTAINING CYCLIC BOOLEAN ALGEBRAS
}

\author{
Peter Rosenthal and A. R. Sourour
}

\begin{abstract}
It is shown that a strongly closed algebra of operators which contains a $\sigma$-complete Boolean algebra with a cyclic vector and which has a totally-ordered invariant subspace lattice must be reflective.
\end{abstract}

1. Introduction. We generalize Arveson's density theorem for transitive algebras containing maximal Abelian von Neumann algebras to the Banach space case; in fact we generalize the totally-ordered version of Arveson's result given in [10]. The main tool (Theorem 7) is a characterization of the linear transformations (not necessarily bounded) which commute with the uniformly closed algebra generated by a Boolean algebra of projections with a cyclic vector. This characterization requires several deep results of Bade on Boolean algebras of projections. In what follows $X$ will be a complex Banach space with dual space $X^{*}$ and $\mathscr{B}(X)$ will be the algebra of all bounded operators on $X$. A subspace means a closed linear manifold.

2. Some properties of cyclic Boolean algebras. Throughout the following $\mathscr{B}$ will denote a Boolean algebra of projections on the Banach space $X$. Recall that $\mathscr{B}$ is $\sigma$-complete if $\left\{E_{n}\right\}_{n=0}^{\infty} \subset \mathscr{B}$ implies that $\mathscr{B}$ contains projections onto $\bigcap_{n=0}^{\infty} E_{n} X$ and onto the closed linear span of $\left\{E_{n} X: n=0,1,2, \cdots\right\}$. A vector $x_{0} \in X$ is cyclic for $\mathscr{B}$ if the closed linear span of $\left\{E x_{0}: E \in \mathscr{B}\right\}$ is all of $X$, and $\mathscr{B}$ is called cyclic if $\mathscr{B}$ has a cyclic vector.

The first three lemmas are due to W. G. Bade ([3]).

LEMMA 1 ([8], p. 2205). If $\mathscr{B}$ is a $\sigma$-complete Boolean algebra of projections on $X$, then for each $x \in X$ there exists a bounded linear functional $x^{*} \in X^{*}$ such that and

(i) $x^{*} E x \geqq 0$ for all $E \in \mathscr{B}$

(ii) if $x^{*} E x=0$ for some $E \in \mathscr{B}$ then $E x=0$.

LEMMA 2 ([8], p. 2207). If $\mathscr{B}$ is a $\sigma$-complete Boolean algebra of projections on $X$ and if $x_{0}$ is a cyclic vector for $\mathscr{B}$ and $x_{0}^{*}$ is the corresponding linear functional of Lemma 1 , then $\left\{E^{*} x_{0}^{*}: E \in \mathscr{B}\right\}$ is total in $X^{*}$. 
LEMMA 3 ([8], p. 2204). Each $\sigma$-complete Boolean algebra of projections is the range of a regular spectral measure defined on a $\sigma$-field of subsets of a compact space; conversely, the range of each such spectral measure is a $\sigma$-complete Boolean algebra.

The next lemma forms the basis of our approach to the main results below.

LEMMA 4. Let $E(\cdot)$ be a spectral measure on a $\sigma$-field $\Sigma$ of subsets of a compact space, and assume that the vector $x_{0}$ is cyclic for $\{E(\sigma)$ : $\sigma \in \Sigma\}$. Then for each $y \in X$ there is an increasing sequence $\left\{\sigma_{n}\right\}$ of sets in $\Sigma$ and there are corresponding bounded operators $R_{n}$ and $S_{n}$ in the uniformly closed algebra generated by $\{E(\sigma): \sigma \in \Sigma\}$ such that:

(i) $E\left(\bigcup_{n=1}^{\infty} \sigma_{n}\right) y=y$

(ii) $E\left(\sigma_{n}\right) y=R_{n} x_{0}$

(iii) $S_{n} y=E\left(\sigma_{n}\right) x_{0}$

(iv) $R_{n} S_{n}=E\left(\sigma_{n}\right)$.

Proof. Let $x_{0}^{*}$ be the linear functional associated to $x_{0}$ by Lemma 1. Then the measure $\sigma \rightarrow x_{0}^{*} E(\sigma) y$ is absolutely continuous with respect to the measure $\sigma \rightarrow x_{0}^{*} E(\sigma) x_{0}$, so the Radon-Nikodym Theorem implies there is a $\Sigma$-measurable function $g$ such that $x_{0}^{*} E(\sigma) y=\int_{\sigma} g(\lambda) d\left(x_{0}^{*} E(\lambda) x_{0}\right)$ for all $\sigma \in \Sigma$. We define

$$
\sigma_{n}=\{\lambda: 1 / n \leqq|g(\lambda)| \leqq n\},
$$

and

$$
R_{n}=\int_{\sigma_{n}} g(\lambda) d E(\lambda), \quad S_{n}=\int_{\sigma_{n}}(1 / g(\lambda)) d E(\lambda) .
$$

Let $\delta$ denote the complement of $\bigcup_{n=1}^{\infty} \sigma_{n}$. Then $g(\lambda)=0$ for $\lambda \in \delta$, so for each $\sigma \in \Sigma$ we have

$$
\begin{aligned}
\left(E(\sigma)^{*} x_{0}^{*}\right) E(\delta) y & =x_{0}^{*}(E(\sigma) E(\delta)) y \\
& =\int_{\sigma \cap \delta} g(\lambda) d\left(x_{0}^{*} E(\lambda) x_{0}\right)=0 .
\end{aligned}
$$

By Lemma 2, then, $E(\delta) y=0$, and (i) follows.

Now for each $\sigma \in \Sigma$ 


$$
\begin{aligned}
\left(E(\sigma)^{*} x_{0}^{*}\right)\left[E\left(\sigma_{n}\right) y\right] & =\int_{\sigma \cap \sigma_{n}} g(\lambda) d\left(x_{0}^{*} E(\lambda) x_{0}\right) \\
& =x_{0}^{*}\left(E(\sigma) R_{n} x_{0}\right) \\
& =\left(E(\sigma)^{*} x_{0}^{*}\right) R_{n} x_{0} .
\end{aligned}
$$

Lemma 2 again applies, and (ii) holds.

Note that (iv) is trivially true. Applying $S_{n}$ to both sides of (ii) and using (iv) yields (iii), so the lemma is proven.

The next lemma is a special case of the lemma on page 2215 of [8].

LEMMA 5. If $E$ is a regular spectral measure such that $\{E(\cdot)\}$ is cyclic, and if $\left\{\delta_{\alpha}\right\}$ is a collection of measurable sets which is pairwise disjoint, then $E\left(\delta_{\alpha}\right)=0$ for all but countably many $\alpha$.

Proof. Let $x_{0}$ be cyclic for $\{E(\cdot)\}$ and choose $x_{0}^{*}$ as in Lemma 1. Then $x_{0}^{*} E\left(\delta_{\alpha}\right) x_{0} \geqq 0$ for all $\alpha$, and $\sum_{j=1}^{\infty} x_{0}^{*} E\left(\delta_{\alpha_{1}}\right) x_{0} \leqq x_{0}^{*} x_{0}$ for all countable subcollections $\left\{\delta_{\alpha_{3}}\right\}$. Hence $x_{0}^{*} E\left(\delta_{\alpha}\right) x_{0}=0$ for all but countably many $\alpha$. Whenever $x^{*} E\left(\delta_{\alpha}\right) x_{0}=0$ then $E\left(\delta_{\alpha}\right) x_{0}=0$ (by Lemma 1 ); $E\left(\delta_{\alpha}\right) x_{0}=0$ implies $E\left(\delta_{\alpha}\right) E(\sigma) x_{0}=0$ for all $\sigma$, and $x_{0}$ cyclic yields $E\left(\delta_{\alpha}\right)=0$.

THEOREM 6. If $\mathscr{B}$ is a $\sigma$-complete cyclic Boolean algebra and $\mathcal{M}$ is an invariant subspace of $\mathscr{B}$, then $\mathscr{M}=E X$ for some $E \in \mathscr{B}$.

Proof. We first show that there is a nonzero $F \in \mathscr{B}$ such that $F X \subset \mathcal{M}$. We can assume $\mathscr{B}$ is a spectral measure, by Lemma 3 . Let $x_{0}$ be cyclic for $\mathscr{B}$, pick $y \neq 0$ in $\mathcal{M}$, and use Lemma 4 to produce a $\sigma_{n}$ such that $E\left(\sigma_{n}\right) y \neq 0$. Then $S_{n} y \in \mathcal{M}$ (since $\mathcal{M}$ is also invariant under the strongly closed algebra generated by $\mathscr{B})$, so $E\left(\sigma_{n}\right) x_{0} \in \mathcal{M}$. Since $x_{0}$ is cyclic it follows that $E\left(\sigma_{n}\right) X \subset \mathcal{M}$, so $F$ can be $E\left(\sigma_{n}\right)$.

Now choose a maximal collection $\left\{\delta_{\alpha}\right\}$ of disjoint sets such that $E\left(\delta_{\alpha}\right) X \subset \mathcal{M}$ and $E\left(\delta_{\alpha}\right) \neq 0$; by Lemma 5 this collection is countable. Let $E=E\left(\cup \delta_{\alpha}\right)$. Clearly $E X \subset \mathcal{M}$. Suppose that $(I-E) \mathcal{M} \neq\{0\}$. Then $(I-E) \mathcal{M}$ is an invariant subspace of $\mathscr{B}$, so by the first paragraph of this proof there is an $F \in \mathscr{B}$ with $F \neq 0$ and $F X \subset$ $(I-E) \mathcal{M}$. Then $F=E(\delta)$ for some $\delta$ disjoint from $\cup \delta_{\alpha}$ and $E(\delta) X \subset$ $\mathcal{M}$, contradicting the maximality of $\left\{\delta_{\alpha}\right\}$. Hence $(I-E) \mathcal{M}=\{0\}$, or $\mu \subset E X$.

3. Unbounded transformations commuting with cyclic Boolean algebras. Let $T$ be a linear transformation with domain $\mathscr{D}$ (a linear manifold in $X$ ) and range a subset of $X$. We say that 
$T$ commutes with the bounded operator $A$ on $X$ if $A \mathscr{D} \subset \mathscr{D}$ and $A T x=T A x$ for all $x \in \mathscr{D} ; T$ commutes with the collection $\mathscr{S}$ of bounded operators if it commutes with each operator in $\mathscr{S}$.

THEOREM 7. Let T be a densely-defined linear transformation which commutes with the uniformly closed algebra generated by the $\sigma$-complete cyclic Boolean algebra $\mathscr{B}$. Then $T$ is closable, and its closure is a scalar-type spectral operator with the range of its resolution of the identity contained in $\mathscr{B}$.

Proof. By Lemma 3, $\mathscr{B}$ is the range of a spectral measure defined on a $\sigma$-field $\Sigma$. Let $x_{0}$ be a cyclic vector for $\mathscr{B}$ and let $x_{0}^{*}$ be the corresponding linear functional given by Lemma 1. Denote the domain of $T$ by $\mathscr{D}$; $\mathscr{D}$ is invariant under all operators in the uniformly closed algebra generated by $\mathscr{B}$.

Choose a maximal collection $\left\{\delta_{\alpha}\right\}$ of disjoint sets such that $E\left(\delta_{\alpha}\right) x_{0} \in$ $\mathscr{D}$ for all $\alpha$. By Lemma 5 at most countably many of the $E\left(\delta_{\alpha}\right)$ are different from 0 ; re-label them $\left\{\delta_{n}\right\}$, and let $\Delta=\cup \delta_{n}$; (if there are no such $\delta_{n}$, then take $\Delta=\varnothing$ ).

We claim that $E(\Delta)=1$. Now if $I-E(\Delta) \neq 0$, choose $z \in \mathscr{D}$ such that $(I-E(\Delta)) z \neq 0$. Apply Lemma 4 to $y=(I-E(\Delta)) z$. Then for $n$ sufficiently large $E\left(\sigma_{n}\right) x_{0} \neq 0$, and $E\left(\sigma_{n}\right) x_{0}=S_{n}(I-E(\Delta)) z \in \mathscr{D}$. Clearly $E(\Delta) E\left(\sigma_{n}\right) E x=0$ for all $E \in \mathscr{B}$, or $E(\Delta) E\left(\sigma_{n}\right)=0$, so, within a set of measure zero, $\sigma_{n}$ is contained in the complement of $\Delta$. This contradicts the maximality of $\left\{\delta_{n}\right\}$, so $E(\Delta)=I$.

For each fixed $n$ the Radon-Nikodym Theorem yields a $\Sigma$ measurable complex-valued function $f_{n}$ such that

$$
x_{0}^{*} T E(\sigma) E\left(\delta_{n}\right) x_{0}=\int_{\sigma} f_{n}(\lambda) d\left(x_{0}^{*} E(\lambda) x_{0}\right)
$$

for all $\sigma \in \Sigma$. Clearly $f_{n}(\lambda)=0$ a.e. when $\lambda \notin \delta_{n}$. Set $f(\lambda)=\sum_{n=1}^{\infty} f_{n}(\lambda)$, define $\tilde{T}=\int f(\lambda) d E(\lambda)$ and let $\tilde{\mathscr{D}}$ be the domain of $\tilde{T}$; $\tilde{T}$ is a closed scalar-type spectral operator (cf. [8], p. 2238). We prove that $\mathscr{D} \subset \tilde{D}$ and $T=\tilde{T} \mid \mathscr{D}$, and then show that $\tilde{T}$ is the closure of $T$.

For each integer $n$ define $\alpha_{n}=\left(\bigcup_{j=1}^{n} \delta_{j}\right) \cap\{\lambda:|f(\lambda)| \leqq n\}$, so that $\left\{\alpha_{n}\right\}$ is an increasing sequence of sets with union $\Delta$. Clearly $E\left(\alpha_{n}\right) X \subset$ $\tilde{\mathscr{D}}$, and for any $\sigma \in \Sigma$ we have

$$
\begin{aligned}
E(\delta)^{*} x_{0}^{*} T E\left(\alpha_{n}\right) E(\sigma) x_{0} & =\int_{\delta \cap \sigma \cap \alpha_{n}} f(\lambda) d\left(x_{0}^{*} E(\lambda) x_{0}\right) \\
& =E(\delta)^{*} x_{0}^{*} \tilde{T} E\left(\alpha_{n}\right) E(\sigma) x_{0} .
\end{aligned}
$$


Hence $T E\left(\alpha_{n}\right)$ and $\tilde{T} E\left(\alpha_{n}\right)$ agree on all vectors of the form $E(\sigma) x_{0}$.

Now fix $y \in D$; we must show that $y \in \tilde{D}$ and $T y=\tilde{T} y$. For each $m$ choose a $\sigma_{m}$ and an $R_{m}$ as in Lemma 4 . For each $m$ and $n$, then,

$$
\begin{aligned}
E\left(\sigma_{m}\right) T E\left(\alpha_{n}\right) y & =T E\left(\alpha_{n}\right) E\left(\sigma_{m}\right) y \\
& =T E\left(\alpha_{n}\right) R_{m} E\left(\sigma_{m}\right) x_{0} \\
& =R_{m} T E\left(\alpha_{n}\right) E\left(\sigma_{m}\right) x_{0} \\
& =R_{m} \tilde{T} E\left(\alpha_{n}\right) E\left(\sigma_{m}\right) x_{0} ; \quad \text { (as shown above, } \\
\tilde{T} E\left(\alpha_{n}\right) & \left.=T E\left(\alpha_{n}\right) \quad \text { on all vectors } E(\sigma) x_{0}\right) .
\end{aligned}
$$

Hence

$$
\begin{aligned}
E\left(\sigma_{m}\right) T E\left(\alpha_{n}\right) y & =\tilde{T} E\left(\alpha_{n}\right) E\left(\sigma_{m}\right) R_{m} x_{0} \\
& =E\left(\sigma_{m}\right) \tilde{T} E\left(\alpha_{n}\right) y
\end{aligned}
$$

Thus

or

$$
E\left(\bigcup_{n=1}^{\infty} \sigma_{m}\right) T E\left(\alpha_{m}\right) y=E\left(\bigcup_{n=1}^{\infty} \sigma_{m}\right) \tilde{T} E\left(\alpha_{n}\right) y
$$

$$
T E\left(\alpha_{n}\right) y=\tilde{T} E\left(\alpha_{n}\right) y,
$$

(since $\left.E\left(\bigcup_{n=1}^{\infty} \sigma_{n}\right) y=y\right)$.

Now $\lim _{n \rightarrow \infty} E\left(\alpha_{n}\right)=I$ and $E\left(\alpha_{n}\right) X \subset \tilde{\mathscr{D}}$, so $\lim _{n \rightarrow \infty} E\left(\alpha_{n}\right) y=y$, and $\lim _{n \rightarrow \infty} \tilde{T} E\left(\alpha_{n}\right) y=\lim _{n \rightarrow \infty} T E\left(\alpha_{n}\right) y=\lim _{n \rightarrow \infty} E\left(\alpha_{n}\right) T y=T y$. Therefore, $y \in \tilde{\mathscr{D}}$ and $\tilde{T} y=T y$.

Now let $\bar{T}$ denote the closure of $T$. Clearly $\bar{T} \subset \tilde{T}$, and we must show equality.

For each $n, E\left(\alpha_{n}\right) X$ is contained in the domain of $\bar{T}$, for $E\left(\alpha_{n}\right) E(\sigma) x_{0}$ is in the domain of $T$ for each $\sigma$ and $T \mid E\left(\alpha_{n}\right) X$ is densely-defined and bounded. Also $E\left(\alpha_{n}\right) X \subset \tilde{\mathscr{D}}$. For $z \in \tilde{\mathscr{D}}$, $\bar{T} E\left(\alpha_{n}\right) z=\tilde{T} E\left(\alpha_{n}\right) z=E\left(\alpha_{n}\right) \tilde{T} z$. Thus $\left\{E\left(\alpha_{n}\right) z \oplus \bar{T} E\left(\alpha_{n}\right) z\right\}$ converges to $z \oplus \tilde{T} z$ as $n$ approaches $\infty$, and $z$ is in the domain of $\bar{T}$. This proves that $\bar{T}=\tilde{T}$.

\section{Arveson's density theorem on Banach} spaces. Recall that a transitive algebra is a subalgebra of $\mathscr{B}(X)$ with no invariant subspaces other than $\{0\}$ and $X$; (see Chapter 8 of [9] for a discussion of this topic). Arveson [1] initiated the study of transitive algebras with his proof of the following theorem in the case where $X$ is a Hilbert space. 
THEOREM 8. If $\mathscr{A}$ is a transitive subalgebra of $\mathscr{B}(X)$ and $\mathscr{A}$ contains a $\sigma$-complete cyclic Boolean algebra, then $\mathscr{A}$ is strongly dense in $\mathscr{B}(X)$.

Proof. Let $\overline{\mathscr{A}}$ denote the strong closure of $\mathscr{A}$. By Arveson's lemma ([9], p. 143) it suffices to show that the only densely-defined linear transformations commuting with $\overline{\mathscr{A}}$ are multiples of the identity; (note that the proof of Arveson's lemma applies without change on Banach spaces). But if $T$ is any such linear transformation then $T$ is closable (by Theorem 7) and its closure $\bar{T}$ is a scalar-type operator commuting with $\mathscr{A}$. If $\bar{T}$ were not a multiple of the identity then $\bar{T}$ would have a nontrivial spectral projection $E$. For each $A \in \mathscr{A}$, then, $A E=E A$, ([8], p. 2229), so the range of $E$ would be a nontrivial invariant subspace of $\mathscr{A}$. Since $\mathscr{A}$ is transitive, $\bar{T}$, and thus also $T$, must be a multiple of the identity.

COROllary 9. If $\mathscr{A}$ is a transitive algebra which contains a scalartype operator $A$ such that $A$ has a cyclic vector and the spectrum of $A$ is nowhere dense and has connected complement, then $\mathscr{A}$ is strongly dense.

Proof. By the result of Dowson [7], the strongly closed algebra generated by $A$ contains the spectral measure $E(\lambda)$ of $A$. Moreover, since the span of $\left\{A^{n} x\right\}$ is clearly contained in the closed linear span of $\{E(\sigma) x: \sigma \in \Sigma\}, E(\lambda)$ is cyclic. Hence the strong closure of $\mathscr{A}$ satisfies the hypotheses of Theorem 8 . in [6].

The theory of Hermitian operators on Banach spaces is treated

Corollary 10 . If $\mathscr{A}$ is a transitive subalgebra of $\mathscr{B}(X)$ with $X$ weakly complete, and if $\mathscr{A}$ contains a cyclic operator all of whose powers are Hermitian, then $\mathscr{A}$ is strongly dense.

Proof. By a theorem of Berkson's [4] such a Hermitian operator is scalar-type, so the result follows from Corollary 9.

There is a general question about transitive algebras on Banach spaces which seems interesting. Given a Banach space $X$ let $\mathscr{H}(X)$ denote the Hermitian operators on $X$. The question is: if $\mathscr{A}$ is a transitive subalgebra of $\mathscr{B}(X)$ which contains $\mathscr{H}(X)$ must $\mathscr{A}$ be strongly dense? If $X$ is Hilbert space then $\mathscr{H}(X)+i \mathscr{H}(X)=\mathscr{B}(X)$, so $\mathscr{A}$ is obviously $\mathscr{B}(X)$. Corollary 10 shows that the answer to this question is affirmative if $X$ is weakly complete and $\mathscr{B}(X)$ contains a cyclic operator whose powers are all Hermitian. In particular, the answer is affirmative when $X$ is a separable $L^{p}(\mu)$ space for $1 \leqq p<\infty$. On the other hand, 
there are Banach spaces $X$ with $\mathscr{H}(X)=\{\lambda I: \lambda \in \mathbf{R}\}$ (cf. [5]); on such spaces the above question reduces to the transitive algebra problem. We can answer the question in one other case.

THEOREM 11. If $X$ is a $C^{*}$-algebra and $\mathscr{A}$ is a transitive subalgebra of $\mathscr{B}(X)$ which contains $\mathscr{H}(X)$, then $\mathscr{A}$ is strongly dense.

Proof. Right and left multiplication by a Hermitian element of $X$ is in $\mathscr{H}(X)$; (in fact, Sinclair [13] shows that $\mathscr{H}(X)$ consists of sums of such operators and ${ }^{*}$-derivations when $X$ is a $C^{*}$-algebra). Since every member of $X$ is the sum of a Hermitian member and $i$ times a Hermitian member, $\mathscr{A}$ contains left and right multiplications by all elements of $X$. Hence the result follows from [12], (where it is observed to be a trivial consequence of the Rickart-Yood Theorem).

\section{Reflexivity of algebras containing cyclic Boolean} algebras. A subalgebra of $\mathscr{B}(X)$ is reflexive if it contains all the operators which leave its invariant subspaces invariant; (see Chapter 9 of [9]). On Hilbert space, Arveson's density theorem has been generalized to show reflexivity of certain algebras whose invariant subspace lattices are totally-ordered ([10]) or complemented ([11]) or, more generally, have finite width ([12]). We show that the first of these generalizations holds on Banach spaces too.

THEOREM 12. If $\mathscr{A}$ is a strongly closed subalgebra of $\mathscr{B}(X)$ which contains a $\sigma$-complete cyclic Boolean algebra, and if the invariant subspaces of $\mathscr{A}$ are totally-ordered, then $\mathscr{A}$ is reflexive.

Proof. We follow the proof of the Hilbert space case as given in [9], beginning on page 181. An examination of this proof shows that it depends on the several properties of $\mathscr{A}$ which we now list and prove.

(i) Every invariant subspace of $\mathscr{A}$ is the range of a projection in $\mathscr{A}$.

Proof. This follows from Theorem 6 above.

(ii) If $T$ is any linear transformation commuting with $\mathscr{A}$, then there is a projection $P \in \mathscr{A}$ such that the domain of $T$ is a dense subset of $P X$ and $T$ is a multiple of the identity on this domain.

Proof. The closure of the domain of $T$ is invariant under $\mathscr{A}$, so by (i) there is a projection $P \in \mathscr{A}$ whose range is the closure of the domain of $T$. For $x$ in the domain of $T,(1-P) T x=T(1-P) x=0$, so $T P X \subset P X$. The restriction of the Boolean algebra to $P X$ is again $\sigma$-complete, ([8], p. 2204), so Theorem 7 implies that the closure of $T \mid P X$ is a scalar-type operator. If $T$ is not a multiple of the identity 
then $T$ has a nontrivial spectral projection $E$ which commutes with $A \mid P X ;$ ([8], p. 2229). Pick nonzero vectors $y=P E y$ and $x=$ $(I-E) P x$, and continuous linear functionals $\phi_{x}$ and $\phi_{y}$ such that $\phi_{x}(x)=1, \phi_{x}(P E X)=\{0\}$ and $\phi_{y}(y)=1, \phi_{y}(P(I-E) X)=\{0\}$. Then, for $A \in \mathscr{A}, \phi_{x}(A y)=\phi_{x}(A P E y)=\phi_{x}(P A P E y)=\phi_{x}(P E A P y)=0$, so the invariant subspace of $\mathscr{A}$ generated by $y$ does not contain $x$. Similarly, for any $A \in \mathscr{A}$

$$
\phi_{y}(A x)=\phi_{y}(A(I-E) P x)=\phi_{y}(P(I-E) A P x)=0,
$$

so the invariant subspace of $\mathscr{A}$ generated by $x$ does not contain $y$. Hence the invariant subspaces of $\mathscr{A}$ generated by $x$ and $y$ are not comparable; this contradiction shows that $T \mid P X$ is a multiple of the identity.

(iii) If $P$ is a projection whose range is invariant under $\mathscr{A}$, then $\tilde{A}=\{(I-P) A \mid(I-P) X: A \in \mathscr{A}\}$ satisfies the hypotheses of the theorem.

Proof. As shown above, $(I-P) \in \mathscr{A}$ and the restriction of the Boolean algebra to $(I-P) X$ is again $\sigma$-complete. If $x_{0}$ is cyclic for the Boolean algebra then $(I-P) x_{0}$ is cyclic for the restriction. Now $\tilde{\mathscr{A}}$ is clearly strongly closed. If $\mathcal{M}$ and $\mathcal{N}$ were noncomparable invariant subspaces of $\tilde{A}$, then $P X+\mathcal{M}$ and $P X+\mathcal{N}$ would be noncomparable invariant subspaces of $\mathscr{A}$. Hence (iii) is proven.

Given (i), (ii), and (iii), the proof of the Hilbert space case given on pages 181-183 of [9] can be followed verbatim.

The following example is given in [10] (and in [9], p. 184) in the case $p=2$.

ExAmPlE 13. If $1 \leqq p<\infty$ and $V$ and $M$ are the operators defined on $L^{p}(0,1)$ by $(V f)(x)=\int_{0}^{x} f(x) d t$ and $(M f)(x)=x f(x)$, then the strongly closed algebra generated by $\{V, M\}$ is the set of all operators $A$ such that, for each $\alpha \in(0,1),(A f)(x)=0$ a.e. on $[0, \alpha]$ whenever $f(x)=0$ a.e. on $[0, \alpha]$.

Proof. The operator $M$ is scalar-type, and its resolution of the identity is cyclic and contained in the strongly closed algebra generated by $M$. Let $\mathscr{A}$ denote the strongly closed algebra generated by $\{V, M\}$. It is easily seen that the invariant subspaces of $\{V, M\}$ are the subspaces of the form

$$
\{f: f=0 \text { a.e. on }[0, \alpha]\}
$$

for $\alpha \in[0,1]$. Hence the result follows from Theorem 12 . 
EXAmple 14. Fix $p$ with $1 \leqq p<\infty$. Define $\mathcal{M}_{n}=\left\{\left\{\alpha_{k}\right\} \in l^{p}\right.$ : $\alpha_{k}=0$ for $\left.k>n\right\}$, and say that $A \in \mathscr{B}\left(l^{p}\right)$ is upper triangular if $A \mathscr{M}_{n} \subset \mathcal{M}_{n}$ for all $n$. Let $\left\{d_{k}\right\}$ and $\left\{w_{k}\right\}$ be bounded sequences of nonzero complex numbers with $\left\{d_{k}\right\} \subset \mathbf{R}$ and $\left\{d_{k}\right\}$ distinct, and define $D\left\{\alpha_{k}\right\}=\left\{d_{k} \alpha_{k}\right\}$ and $W\left\{\alpha_{k}\right\}=\left\{w_{k} \alpha_{k+1}\right\}$. Then the trianglular operators are the operators in the strongly closed algebra generated by $D$ and $W$.

Proof. This can be shown by direct computations. Alternatively, let $\mathscr{A}$ denote the strongly closed algebra generated by $D$ and $W$. Then the nontrivial invariant subspaces of $\mathscr{A}$ are $\left\{\mathcal{M}_{n}\right\}$ and $\mathscr{A}$ contains the $\sigma$-complete Boolean algebra consisting of the diagonal operators with diagonal elements 0 or 1 . Hence Theorem 12 applies and $\mathscr{A}$ is reflexive.

Note that Example 14 also holds on the space $c_{0}$.

E. Azoff has suggested the following question. If $\mathscr{A}$ is a strongly closed subalgebra of $\mathscr{B}(X)$ and every invariant subspace of $\mathscr{A}$ has an invariant complement, must $\mathscr{A}$ be reflexive? An affirmative answer would obviously imply that every reductive algebra on Hilbert space is self-adjoint; (cf. [9], p. 167). The above technique might lead to an affirmative answer to Azoff's question in the case where $\mathscr{A}$ contains a self-adjoint; (cf. [9], p. 167). The above techniques might lead to an affirmative answer to Azoff's question in the case where $\mathscr{A}$ contains a cyclic Boolean algebra, (following the proof of and generalizing [11]).

Note added in proof. The question of Azoff mentioned in the preceding paragraph has been shown to have an affirmative answer in the case where the algebra $\mathscr{A}$ contains a cyclic Boolean algebra. Also, Theorems 6, 7 and 8 hold when $\beta$ is a Boolean algebra of multiplicity one in the sense of Bade. These results can be found in the author's "On operator algebras containing cyclic Boolean algebras II", J. London Math. Soc., to appear.

\section{REFERENCES}

1. W. B. Arveson, A density theorem for operator algebras, Duke Math. J., 34 (1967), 635-647.

2. - Operator algebras and invarlant subspaces. Ann. of Math., (2) 100 (1974), 433-532.

3. W. G. Bade, On Boolean algebras of projections and algebras of operators, Trans. Amer. Math. Soc., 80 (1955), 345-360).

4. Earl Berkson, A characterization of scalar-type operators on reflexive Banach spaces, Pacific $\mathrm{J}$. Math., 13 (1963), 365-373.

5. Earl Berkson and Ahmed Sourour, The hermitian operators on some Banach spaces, Studia Math., 52 (1974), 33-41.

6. F. F. Bonsall and J. Duncan, Numerical Ranges I and II, London, Cambridge University Press, 1971 and 1973.

7. H. R. Dowson, On some algebras of operators generated by a scalar-type spectral operator, J. London Math. Soc., 40 (1965), 589-593.

8. N. Dunford and J. T. Schwartz, Linear Operators Part III, New York, Wiley-Interscience, 1971. 
9. H. Radjavi and P. Rosenthal, Invariant Subspaces, Berlin, Springer-Verlag, 1973.

10. - On invariant subspaces and reflexive algebras, Amer. J. Math., 91 (1969), 683-692.

11. —_, A sufficient condition that an operator algebra be self-adjoint, Canad. J. Math., 23 (1971), 588-597.

12. - On transitive and reductive operator algebras, Math. Annalen, 209 (1974), 43-56.

13. A. Sinclair, Jordan homomorphisms and derivations on semisimple Banach algebras, Proc. Amer. Math. Soc., 24 (1970), 209-214.

Received February 18, 1976.

University of Toronto, Ontario, CANADA 



\section{Pacific Journal of Mathematics \\ Vol. 70, No. $1 \quad$ September, 1977}

William H. Barker, Noether's theorem for plane domains with hyperelliptic

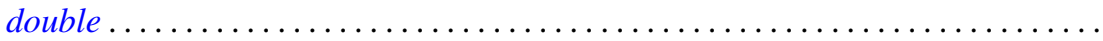

Michael James Beeson, Non-continuous dependence of surfaces of least area on the boundary curve ...................................... 11

Horst Behncke, Functions acting in weighted Orlicz algebras . . . . . . . . . . . . 19

Howard Edwin Bell, A commutativity study for periodic rings . . . . . . . . . . . 29

Peter Botta and Stephen J. Pierce, The preservers of any orthogonal group ....... 37

Douglas S. Bridges, The constructive Radon-Nikodým theorem ............. 51

James Dennis Brom, The theory of almost periodic functions in constructive

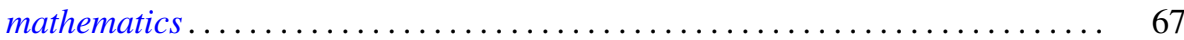

N. Burgoyne and C. Williamson, Semi-simple classes in Chevalley type groups ....

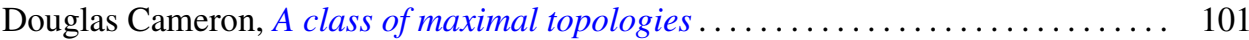

L. Carlitz, Enumeration of doubly up-down permutations . . . . . . . . . . . . . . 105

Paul Robert Chernoff, The quantum n-body problem and a theorem of

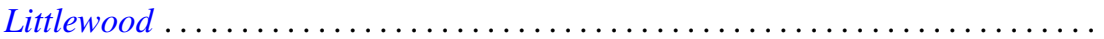

Jo-Ann Deborah Cohen, Locally bounded topologies on $F(X) \ldots \ldots \ldots \ldots \ldots \ldots$

Heinz Otto Cordes and Robert Colman McOwen, Remarks on singular elliptic

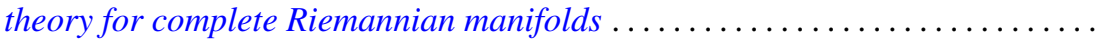

Micheal Neal Dyer, Correction to: "Rational homology and Whitehead

products"

Robert Fernholz, Factorization of Radonifying transformations

Lawrence Arthur Fialkow, A note on quasisimilarity. II ...... . .

Harvey Charles Greenwald, Lipschitz spaces of distributions on the surface of unit

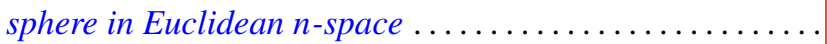

Albrecht Irle, On the measurability of conditional expectations

Tom (Roy Thomas Jr.) Jacob, Matrix transformations involving simple sequence

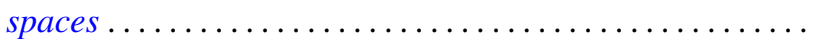

A. Katsaras, Continuous linear maps positive on increasing continuous

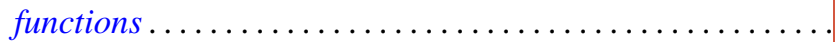

Kenneth Kunen and Judith Roitman, Attaining the spread at cardinals of cofinality

Lawrence Louis Larmore and Robert David Rigdon, Enumerating normal bundles

of immersions and embeddings of projective spaces ...... . .

Ch. G. Philos and V. A. Staïkos, Asymptotic properties of nonoscillatory solutions of differential equations with deviating argument .

Peter Michael Rosenthal and Ahmed Ramzy Sourour, On operator algebras containing cyclic Boolean algebras...

Polychronis Strantzalos, Strikt fast gleichgradig-stetige und eigentliche

Aktionen ...

Glenn Francis Webb, Exponential representation of solutions to an abstract

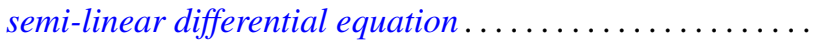

